\title{
Gross Shell Structure Of Moments Of Inertia
}

\author{
M.A. Deleplanque*, S. Frauendorf ${ }^{\dagger}$, V.V. Pashkevich ${ }^{* *}$, S.Y. Chu* and \\ A. Unzhakova** \\ *Nuclear Science Division, Lawrence Berkeley National Laboratory, Berkeley, California, 94720 \\ ${ }^{\dagger}$ Physics Department, University of Notre Dame, Notre Dame, IN 46556 \\ ${ }^{* *}$ Joint Institute of Nuclear Research, Dubna, Russia
}

\begin{abstract}
Average yrast moments of inertia at high spins, where the pairing correlations are expected to be largely absent, were found to deviate from the rigid-body values. This indicates that shell effects contribute to the moment of inertia. We discuss the gross dependence of moments of inertia and shell energies on the neutron number in terms of the semiclassical periodic orbit theory. We show that the ground-state shell energies, nuclear deformations and deviations from rigid-body moments of inertia are all due to the same periodic orbits.
\end{abstract}

\section{INTRODUCTION}

We know from experiments and from microscopic calculations that shell effects, which are the bunching of singleparticle levels in quantal systems, influence strongly the stability and deformation of nuclei. In this talk, I want to study the contribution of shell effects to moments of inertia at high spins along the yrast line where the pairing is largely absent. In this case, if there are no shell effects, the moment of inertia takes the rigid-body value. Therefore, in our study of shell effects, we will be looking for deviation of the moment of inertia from rigid-body values. However, gross shell effects depend only on an averaged or "coarsed-grained" level density; therefore there is no need for full quantization and we use a semiclassical theory: the Periodic Orbit Theory which relates the quantum fluctuations to properties of a (few) periodic orbits in the potential considered. I will show that the gross dependence of the moment of inertia on neutron number can be deduced from the properties of these orbits.

\section{EXPERIMENTAL METHOD}

We want to determine an "average" moment of inertia along the yrast line at high spins. The yrast line includes both odd and even spins and parities. We characterize the yrast line by one moment of inertia $\mathscr{J}_{\text {exp }}$ which is deduced from the slope of an energy $\underline{v} \mathrm{I}(\mathrm{I}+1)$ plot, where $\mathrm{I}$ is the level spin.

$\mathscr{J}_{\exp }$ is given by:

$$
E(I)=E_{0}+\frac{\vec{h}}{2 \mathscr{J}_{\text {exp }}} I(I+1)
$$

where the slope is fitted at the highest spins, as shown in Fig. 1.

The curvature at low spins is due to the pairing correlations which, as is well known, reduce the moment of inertia. In our systematic study, however, we are selecting nuclei whose maximum spin is greater than 15 and usually much higher. In the large majority of cases, the curve is linear at the highest spins and we consider that the pairing correlations are largely absent. The deviation from the rigid-body value is then:

$$
\mathscr{J}_{s h}=\mathscr{J}_{\text {exp }}-\mathscr{J}_{\text {rig }}
$$

$\mathscr{J}_{s h}$ is the shell contribution to the moment of inertia.

The data are extracted from the ENSDF data base for nuclear mass numbers A 80 to 220, using the "Isotope Explorer" [1] program. For $\mathscr{J}_{\text {rig }}$ we calculate the value which corresponds to a rotation perpendicular to the symmetry axis 

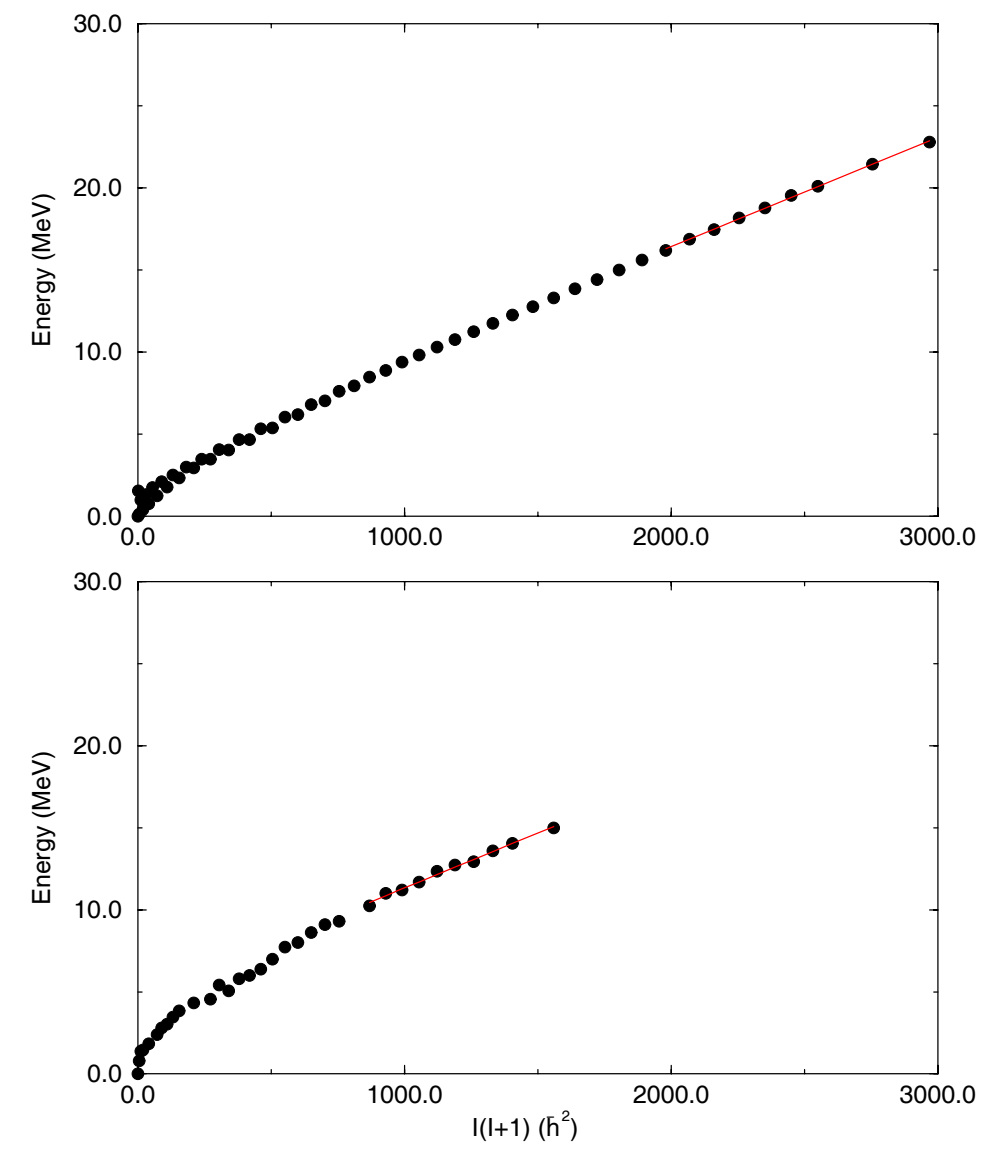

FIGURE 1. Yrast lines for a rotational nucleus ${ }^{160} \operatorname{Er}$ (top) and for a non-rotational nucleus ${ }^{150} D y$ (bottom) together with their fit for the ten highest spins.

which is practically always the case. The deformation $\beta$ is taken to be the ground state deformation as given by Möller [2]. Since the moment of inertia is quite insensitive to the deformation, this is a good enough approximation. $\mathscr{J}_{\text {rig }}$ is given by:

$$
\mathscr{J}_{\text {rig }}=0.01253 A^{5 / 3}(1+1 / 2 \sqrt{5 / 4 \pi} \beta)+0.048225 A
$$

\section{EXPERIMENTAL RESULTS}

Fig. 2 shows a plot of the deviations $\mathscr{J}_{s h}$ of the experimental moments of inertia from rigid body, plotted as a function of neutron number for a series of proton numbers labeled by the symbols. One sees that there are large deviations from rigid-body values, reaching $-65 \%$ around neutron number 126 . In this talk, we will analyze mainly the structure between neutron numbers 82 and 126 where the effects are clearer because the proton and neutron shells are more in phase. For a more detailed analysis, we refer to an upcoming paper [3].

To gain more insight, we compare these deviations to the experimental ground-state shell energies $E_{s h}$ which are shown in Fig. 3. These shell energies are obtained from the experimental ground-state masses in ref [2] by subtracting a macroscopic energy $E_{L D}$ calculated also in ref [2] and correcting for deformation as prescribed by W. Swiatecki [4]. We see that the variations of ground-state shell energies and shell moments of inertia as a function of neutron number 


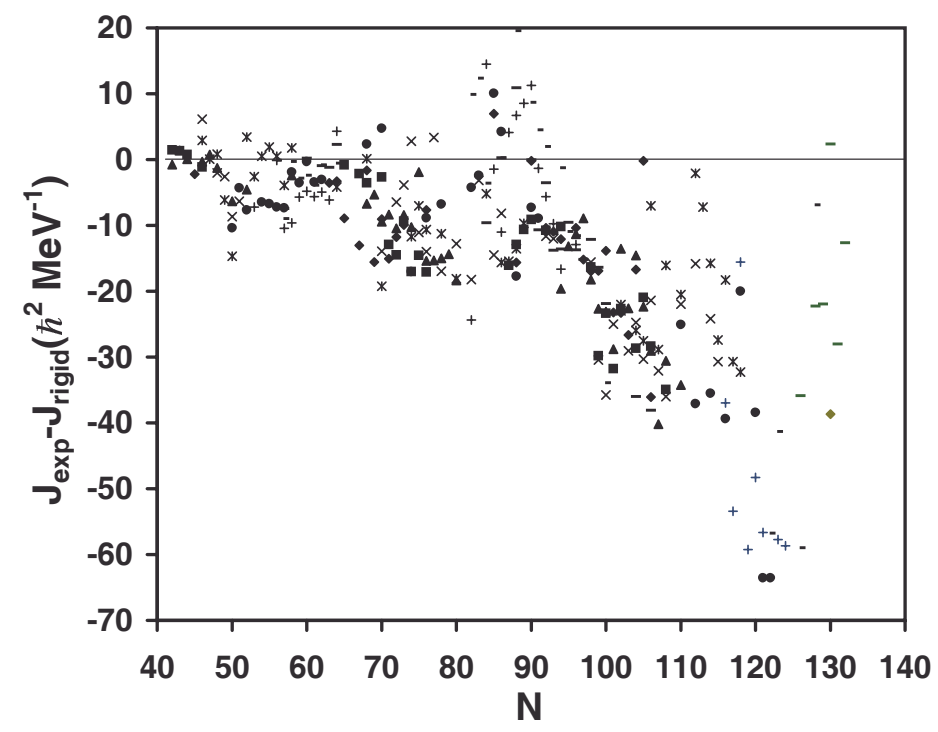

FIGURE 2. Experimental deviations from the rigid-body moments of inertia as a function of neutron number for small and normal deformation. The proton symbols are the same as in Fig. 3.

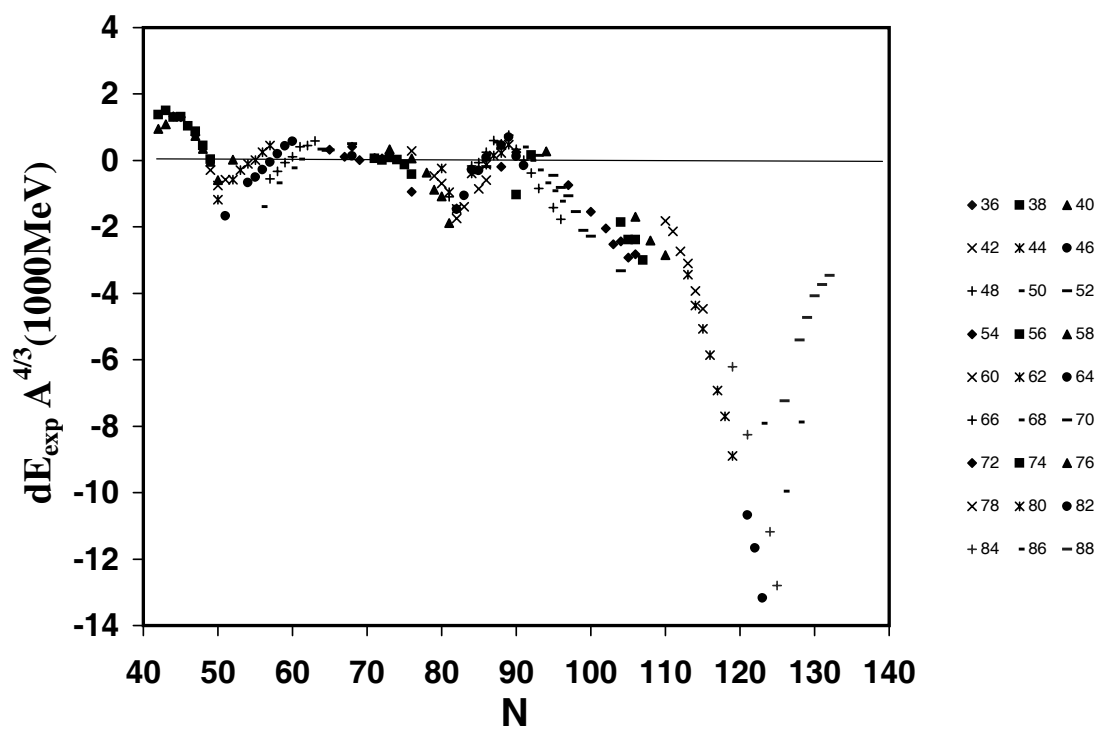

FIGURE 3. Experimental shell energies at spin 0 as a function of neutron number.

have strong similarities: there are pronounced minima at the spherical magic numbers and oscillations between those.

We note particularly a positive "bump" around neutron number 90 and another bump around neutron number 110 . The latter corresponds to the region of high-spin isomers and is perhaps more pronounced in the shell moment of inertia plot. I want to show you that this similarity means that the same "periodic orbits" produce the shell structure in both $E_{s h}$ and $\mathscr{J}_{s h}$. Notice that we have actually plotted the quantity $E_{s h} A^{4 / 3}$ in Fig. 3. The reason will become clearer later in this talk. 


\section{INTERPRETATION}

As I said before, we are only interested in gross shell structure and in that case it is sufficient to look at the important periodic orbits which are identified in the Periodic Orbit Theory (POT). I will therefore give a quick reminder of what we know from POT and go on to apply it to the case of a rotating nucleus.

\section{Basic Elements Of POT}

In this theory, the nucleus is generally considered as a hollow cavity with nucleons moving at constant velocities along straight lines in periodic orbits with perfect reflection on the cavity walls. This model works because such a system has the main characteristics of a Fermionic motion in a nucleus, namely independent nucleons bound by a potential that they cannot escape. For a comprehensive introduction to POT, see $[5,6]$.

One of the principal results is that the total energy $E\left(k_{F}\right)$ can be decomposed into a smooth part $\tilde{E}\left(k_{F}\right)$ and a fluctuating part $E_{s h}\left(k_{F}\right)$ which accounts for the quantum (or shell) effects. This is expressed in the following two equations:

$$
\begin{gathered}
E\left(k_{F}\right)=\tilde{E}\left(k_{F}\right)+E_{s h}\left(k_{F}\right) \\
E_{s h}\left(k_{F}\right)=\sum_{\beta} \mathscr{A}_{\beta}\left(k_{F}\right) \sin \left(L_{\beta} k_{F}+v_{\beta}\right) D_{\beta}\left(\gamma, L_{\beta}\right)
\end{gathered}
$$

where $E_{s h}\left(k_{F}\right)$ is a sum of periodic functions. $k_{F}$ is the Fermi wave number, and $L_{\beta}$ is the length of the orbits in the family $\beta . L_{\beta} k_{F}$ is the action of the nucleons on these orbits. The factor $\mathscr{A}_{\beta}\left(k_{F}\right)$ determines the contribution from each family and depends mainly on the degeneracy and stability of the orbits. I want to emphasize two points: first, $E_{s h}\left(k_{F}\right)$ is a sum over families of orbits, which means that there can be interference between different orbit families, and second, there is a so-called damping factor $D_{\beta}\left(\gamma, L_{\beta}\right)$ which comes from averaging over a certain energy interval $\gamma$ and suppresses the contribution from the longer orbits.

Thus, if $\gamma$ is smaller than the energy separation of the main spherical shells, the gross shell structure remains. Balian and Bloch [7] were the first to study the spherical cavity and show that the contributing periodic orbits were planar polygons. Averaging over energy, only the shortest orbits of highest degeneracy contribute significantly, i.e., the triangle and the square. The contribution of the diameter, which has a degeneracy of two as compared to three for the triangle and the square, is not important. Thus, in the remainder of this talk, I shall be considering these two orbit families, and often only one of them for simplicity. For example, if one considers only one orbit family, then Eq. (4) is a sine function and the minimum of the shell energy is given by the values of $L_{\beta} k_{F}$ which minimize it. Since $L_{\beta}$ depends on the nucleon number, this condition will yield the spherical magic numbers. In practise, one should consider protons and neutrons separately, but we will not elaborate on this point.

\section{The Deformed Nucleus At Spin Zero}

The deformed nucleus is now modeled by a spheroidal cavity. It is then necessary to consider two different types of orbits (see Fig. 4): (1) the meridian orbits (the triangle and rhomboid) which are in the planes containing the symmetry axis of the spheroid and have a two-fold degeneracy (Fig. 4, bottom), and (2) the equatorial orbits (the triangle and the square) which are in the plane perpendicular to the symmetry axis of the spheroid and have a one-fold degeneracy (Fig. 4, top). V.M. Strutinsky and coworkers [8] were the first to show that, because of their higher degeneracy, the meridian orbits are the main contributors to the shell structure in deformed nuclei. In the same way as for the spherical cavity, if one considers only the rhomboids for example, then the minima of the shell energy, which we now note $E_{s h}(0)$ (for spin 0), will be given by the locus of the nuclei where the orbit lengths are constant and minimize the sine function. However, the lengths of the rhomboids, which we now denote by the $\perp$ sign for reasons that will become clear in the context of rotation, depend on two quantities: the nucleon number and the deformation, which we now label by the ratio $\eta$ of the long axis to short axis. Therefore the stable deformed nuclei will follow the valley of the minima of $E_{s h}(0)$ in the $(N, \eta)$ plane which correspond to a line of constant $L_{\perp}(N, \eta)$. In other words, the structure of $E_{s h}(0)$ in the $(N, \eta)$ plane will follow the oscillations given by the meridian orbits. However, a careful examination 

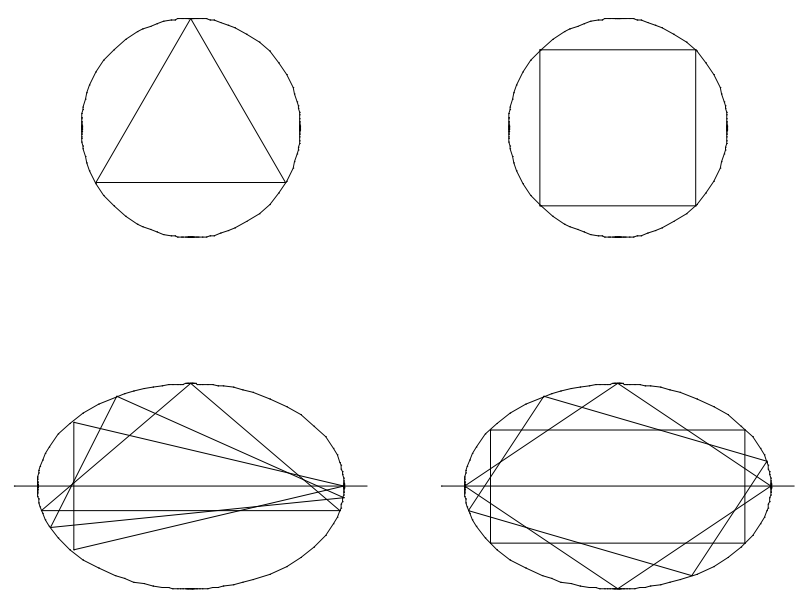

FIGURE 4. Classical periodic orbits in the equator plane (upper panel) and meridian plane (lower panel) of a normal deformed spheroidal cavity.

of this structure reveals an interference pattern which is due to the contribution of the equatorial orbits (they give rise to the bump at $\mathrm{N} \sim 110$ in Figs. 2 and 3). Although less important, these orbits nevertheless play a role in the nuclear shell structure.

\section{Influence Of Rotation}

The problem of symmetry breaking in the POT is complex and has been treated in a general way in the case of a perturbation, see for example the formulation of Creagh [9]. In the case of rotation, the perturbation to the main cavity potential is the rotational term $\omega l$, where $\omega$ is the angular velocity and $l$ is the projection on the rotation axis of the angular momentum of the nucleon on the periodic orbit. Creagh showed that this perturbation's main effect is a change in the action (the phase of the periodic function). Its contribution is the flux $\Phi$ of the vector $2 m \vec{\omega}$ across the area of the periodic orbit considered:

$$
\Phi=2 m \overrightarrow{A_{\beta}} \cdot \vec{\omega}
$$

where $m$ is the nucleon mass and $\overrightarrow{A_{\beta}}$ is a vector perpendicular to the plane of the orbit and whose length is the area enclosed by the periodic orbit. From this, we see that if the flux of $\vec{\omega}$ across a periodic orbit is non-zero, this orbit will have an effect due to rotation and will contribute to the oscillating or shell part of the moment of inertia. Fig. 5 illustrates the effect of meridian and equatorial orbits depending on the rotation axis. For a meridian orbit, if the rotation is parallel to the symmetry axis $\mathrm{z}$ (bottom left), the flux accross the orbit is zero because the area vector, now noted $\overrightarrow{A_{m}}$, is perpendicular to $\vec{\omega}$. Conversely, if the rotation is perpendicular to the symmetry axis (top left), the flux is non-zero and there will be a contribution to $\mathscr{J}_{s h}$. This is why we have labeled the meridian orbits by the subscript $\perp$. Equatorial orbits will contribute to $\mathscr{J}_{s h}$ for a rotation parallel to the symmetry axis (bottom right) and this is why we label them by the subscript $\|$. The top right case corresponds to the situation for superdeformed nuclei. K. Matsuyanagi $[10,6]$ has shown that equatorial orbits are the important ones for superdeformed nuclei. Since we know that these nuclei rotate around an axis perpendicular to the symmetry axis, the flux is zero. Therefore the moment of inertia of these nuclei should be rigid (no shell contribution). We have indeed found that for the superdeformed nuclei with an axis ratio $\eta=2: 1$, the band moments of inertia, which constitute the yrast line at these high spins, deviate on the average from the rigid-body value by only $\sim 3 \%$. K. Matsuyanagi[10] showed that three-dimensional orbits are also 
meridian

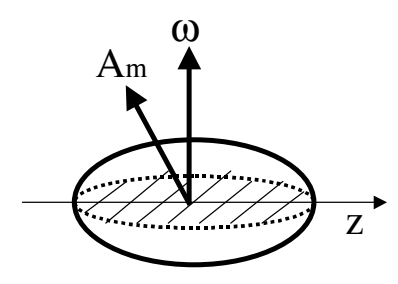

Flux $=2 \mathrm{~m} \overrightarrow{\mathrm{Am}} \cdot \vec{\omega}$

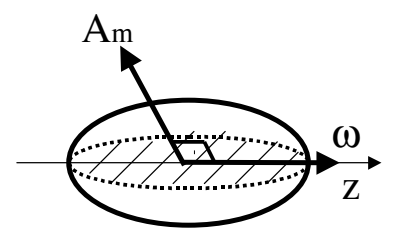

Flux $=0$ equatorial

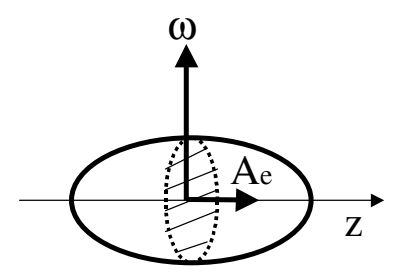

Flux $=0$

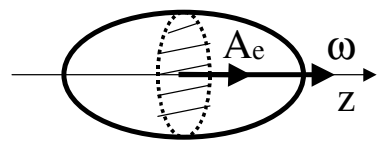

Flux $=2 \mathrm{~m} \mathrm{Ae} . \omega$

FIGURE 5. Flux of the angular velocity vector across meridian (left) and equatorial (right) classical periodic orbits for a nuclear rotation perpendicular (top) and parallel (bottom) to the symmetry axis.

main contributors to the shell structure of superdeformed nuclei. In that case, it is also possible to show that the flux of $\vec{\omega}$ is zero so that the argument given above is valid.

We conclude that since the meridian orbits are the main contributors to the deformed shell structure and since most nuclei rotate around an axis perpendicular to the symmetry axis, there will generally be a shell contribution to the moment of inertia and we will see deviations from the rigid-body values. We shall now express quantitatively this shell contribution and see how the correlation between $\mathscr{J}_{s h}$ and $E_{s h}(0)$ appears.

\section{Shell Moments Of Inertia}

The shell energy $E_{s h}^{\prime}\left(k_{F}, \omega\right)$ at "frequency" $\omega$ is a sum, over the families $\beta$ of periodic orbits that contribute to the moment of inertia, of the shell energy at zero spin times a so-called modulation factor $\mathscr{M}\left(\Phi_{\beta}\right)$ which comes from averaging the fluxes over the periodic orbits in a family. It is expressed by:

$$
E_{s h}^{\prime}\left(k_{F}, \omega\right)=\sum_{\beta} \mathscr{M}\left(\Phi_{\beta}\right) E_{s h \beta}\left(k_{F}, 0\right)
$$

where $\Phi_{\beta}$ is the flux through an orbit perpendicular to the axis of rotation. For low frequencies, the modulation factor can be expanded to second order in $\Phi_{\beta}$ :

$$
\mathscr{M}\left(\Phi_{\beta}\right) \sim 1-a \Phi_{\beta}^{2}
$$

where the coefficient, a, depends on whether the orbit is equatorial or meridian or in a spherical cavity $[11,12,3]$. We see that a term in $\omega^{2}$ appears in the shell energy at frequency $\omega$ and we identify its coefficient with half the shell moment of inertia. Thus we have: 


$$
\begin{gathered}
E_{s h}^{\prime}\left(k_{F}, \omega\right)=E_{s h}\left(k_{F}, 0\right)-\frac{\omega^{2}}{2} \mathscr{J}_{s h}\left(k_{F}, \omega\right) \\
\mathscr{J}_{s h \beta}(\omega)=a \frac{l_{\beta}^{2} \tau_{\beta}^{2}}{\vec{h}^{2}} E_{s h \beta}(0) \sim \frac{1}{b}\left(\frac{\vec{h}}{M e V^{2}}\right) A^{4 / 3} E_{s h \beta}(0)
\end{gathered}
$$

This shows that, for each family $\beta$ which contributes to the shell moment of inertia, $\mathscr{J}_{s h \beta}(\omega)$ is proportional to $A^{4 / 3} E_{s h \beta}(0)$. For the triangle orbits the factor $\mathrm{b}$ is 400 and of the same order for the square. We now see that, if there is only one family of contributing orbits, $\mathscr{J}_{s h}$ is correlated with $E_{s h}(0)$. This is generally true as we have seen that only one kind of orbit (meridian or equatorial) contributes depending on the axis of rotation. But the converse is not necessarily true since both kinds of orbits can contribute to $E_{s h}(0)$ independent of the axis of rotation. Nevertheless, we show now that this simple correlation is generally preserved.

\section{Correlation Between $\mathscr{J}_{s h}$ And $E_{s h}(0)$}

The reason this correlation is preserved is that generally the meridian orbits dominate the shell structure and that the nucleus rotates almost always around an axis perpendicular to the symmetry axis. Thus we have:

$$
\mathscr{J}_{s h}(\omega)=\mathscr{J}_{s h \perp}(\omega) \sim E_{s h \perp}(0) \sim E_{s h}(0)
$$

This accounts for the general similarity of the oscillations in Fig. 2 and 3. We also see that the ratio of the scales in Fig. 2 and 3 is 1/200, differing only by a factor of two from the estimate in Eq. 10. This is remarkably good considering the qualitative character of the arguments given.

However, in Fig. 3, the bump at $N \sim 110$ is due to the equatorial orbits (high-K isomer region). It has a positive contribution, superposed on the generally negative contribution of the meridian orbits. The equatorial orbits give also a strong positive contribution to the moment of inertia for rotation about the symmetry axis. This leads to a preference of rotation about the symmetry axis, which is experimentally observed as the appearance of high- $\mathrm{K}$ isomers in this region. If the yrast line were composed only of isomeric band heads, the experimental shell moment of inertia should become positive. In experiment, this is not the case (Fig. 2) because the yrast line is mostly composed of the high rotational states based on the high-K bandhead. Thus the rotational axis is not the symmetry axis but it is tilted and the moment of inertia has contributions from rotation about the symmetry axis and the axis perpendicular to it. Our quantum mechanical cranking calculations [3] for a Woods-Saxon potential, which rotates about the symmetry axis, show the expected strongly positive values of $\mathscr{J}_{s h}$.

\section{CONCLUSION}

We have shown that the moments of inertia at high spins along the yrast line are not rigid. The shell effects, which produce the deviations from the rigid-body values, contribute to the moments of inertia up to the highest spins observed. We have provided a qualitative interpretation of the shell structure in terms of the Periodic Orbit Theory. We have shown that the ground-state shell energies, the nuclear deformation and the deviations from the rigid-body moments of inertia are all due to the same periodic orbits.

\section{ACKNOWLEDGMENTS}

We would like to thank P. Fallon for providing the data base and analysis program for superdeformed nuclei and R. Firestone for help in constructing figures. This work was supported by the Director, Office of Energy Research, Division of Nuclear Physics of the Office of High Energy and Nuclear Physics of the U.S. Department of Energy under contract No. DE-AC03-76SF00098. 


\section{REFERENCES}

1. Chu, S.Y.F., Ekström, L.P., Firestone, R.B., Isotope Explorer programs available on the WWW at http://ie.lbl.gov/ensdf(JAVA/HTML program) and http://ie.lbl.gov/isoexpl/isoexpl.htm( $C^{++}$program).

2. Möller, P., Nix, J.R., Myers, W.D., and Swiatecki, W.J., Atomic Data and Nuclear Data Tables 59, 185 (1995).

3. Deleplanque, M.A., Frauendorf, S., Pashkevich, V.V., Chu, S.Y.F., and Unzhakova, A., to be submitted to Phys. Rev. C.

4. Swiatecki, W.J., private communication.

5. $\quad$ Brack, M., and Bhaduri, R.K., Semiclassical Physics, Addison-Wesley, 1997.

6. Matsuyanagi, K., this conference.

7. Balian, R., and Bloch, C., Ann. of Phys. 69, 76 (1972).

8. Strutinsky, V.M., Magner, A.G., Ofengenden, S.R., and Døssing, T., Z. Phys. A283, 269 (1977).

9. Creagh, S.C., Ann. of Phys. 248, 60 (1996).

10. Arita, K., Sugita, A., and Matsuyanagi, K., Prog. Th. Phys. 100, 1223 (1998).

11. Kolomiets, V.M., Magner, A.G., and Strutinsky, V.M., Yad. Fiz. 29, 1478 (1979).

12. Frauendorf, S., Kolomiets, V.M., Magner, A.G., and Sanzhur, A.I., Phys. Rev B58, 5622 (1998). 